\title{
Obesity is Associated with Poor Covid-19 Outcomes: A Systematic Review and Meta-Analysis
}

Authors

Caroline Wei Shan Hoong ${ }^{1}$, Ikram Hussain ${ }^{2}$, Veeraraghavan Meyyur Aravamudan ${ }^{3}$, Ei Ei Phyu ${ }^{4}$, Jaime Hui Xian Lin ${ }^{{ }^{*}}$, Huilin Koh ${ }^{*}$

Affiliations

1 Department of Endocrinology, Woodlands Health Campus Singapore, Singapore

2 Department of Gastroenterology, Woodlands Health Campus Singapore, Singapore

3 Department of Medicine, Woodlands Health Campus Singapore, Singapore

4 Department of Geriatric Medicine, William Harvey Hospital, Ashford, Kent, UK

received 10.07.2020

accepted after revision $\quad 22.11 .2020$

published online $\quad 04.01 .2021$

Bibliography

Horm Metab Res 2021; 53: 85-93

DOI 10.1055/a-1326-2125

ISSN 0018-5043

(c) 2021. Thieme. All rights reserved.

Georg Thieme Verlag KG, Rüdigerstraße 14,

70469 Stuttgart, Germany

Correspondence

Caroline Wei Shan Hoong

Department of Endocrinology

Woodlands Health Campus Singapore

National Healthcare Group

Singapore

caroline_hoong@whc.sg
Supplementary material is available under https:// doi.org/10.1055/a-1326-2125.

\section{ABSTRACT}

Our aim was to assess the association between obesity and the risk of unfavourable outcomes (composite of severe disease and mortality) in inpatients with COVID-19. We conducted a systematic search of databases between December 2019 and 28 th June 2020 . Studies were included if they reported or allowed estimation of an odds ratio (OR) for unfavourable outcome in obese compared to non-obese patients hospitalised for COVID-19. Twenty cohort studies of 28355 hospitalised patients with COVID-19 infection were included. Meta-analysis estimated a pooled OR of $2.02(1.41-2.89, \mathrm{p}<0.001)$ for an unfavourable outcome in obese versus non-obese patients when adjusted for age, sex and co-morbidities. When unadjusted for confounders, the OR for unfavourable outcomes was 1.25 ( Cl 1.07-1.45, $\mathrm{p}=0.005)$. An increased adjusted $\mathrm{OR}$ was also seen for death (OR 1.51; $\mathrm{Cl} 1.13-2.21, \mathrm{p}=0.006)$ and severe illness (OR 2.26; $\mathrm{Cl} 1.47-3.48, \mathrm{p}<0.001)$. Compared to a normal BMI, the risk of an unfavourable outcome was increased even in overweight patients, with severe obesity having an escalated risk. Obesity is independently associated with an unfavourable outcome of COVID-19 illness, with obese patients having twice the risk of a composite outcome of severe disease or mortality, and a $50 \%$ increased risk of death.

\section{Introduction}

Since the discovery in December 2019, the novel Coronavirus Disease 2019 (COVID-19) has taken the world by storm with the declaration of a global health emergency on 30th January 2020 by the World Health Organization (WHO) [1]. At the latest count among 213 countries in early July 2020, 11600000 infections and more than 537000 deaths have been reported worldwide with many countries still struggling to control the spread of this contagion [2].

The clinical course of COVID-19 ranges from asymptomatic or mild infection to severe, life-threatening pneumonia with multi-or-

Joint first and last authors. gan failure. With no definitive curative treatment found as yet, public health systems have sought to risk stratify and protect the more vulnerable groups in the population. Several early studies have identified some host-related risk factors, which may predispose to severe illness, such as older age and underlying comorbidities such as chronic respiratory illness, cardiovascular disease (CVD), diabetes mellitus and hypertension [3-6]. This has led to the international health authorities promoting shielding strategies of vulnerable groups of individuals above 60-70 years, the immunocompromised, or those with chronic comorbidities [7-10]. Although not studied during the initial period of COVID-19, increased body-mass index (BMI) has been increasingly reported to carry an independent risk for severe COVID-19 infection [11-14]. 
Studies before the COVID-19 pandemic have been conflicting in terms of the association of obesity with poorer outcomes for acute respiratory illnesses. For severe outcomes of influenza pneumonia including hospitalisation, ICU admissions and death, results have been mixed regarding obesity as a risk factor [15-19]. However, a systematic review and meta-analysis of 234 studies and 610782 participants found that obesity is an independent significant risk factor for severe outcomes in both the seasonal influenza as well as the pandemic H1N1 influenza [20].

With respect to the COVID-19 infection, we seek to elucidate whether obesity confers a poorer prognosis as observed in previous pandemic pneumonias. Hence, we undertook a systematic review and meta-analysis to assess the impact of obesity on unfavourable outcomes of COVID-19 disease in hospitalised adult patients, as compared to non-obese patients.

\section{Materials and Methods}

This systematic review was performed in accordance with the Cochrane Collaboration guidelines [21]. The study followed the guidelines from the Preferred Reporting Items for Systematic Reviews and Meta-Analyses (PRISMA) and the Meta-analyses of Observational Studies in Epidemiology (MOOSE) [22, 23].

\section{Literature search}

Three authors (CWSH, HK, and JHXL) undertook independent comprehensive search for published articles from the MEDLINE, EMBASE, Web of Science, and Cochrane databases. The articles were searched from 1st December 2019 until 28th June 2020. The search terms included 'COVID-19, 'SARS-CoV2', 'coronavirus disease 2019', 'novel coronavirus', 'outcomes', 'obesity', 'body mass index', and 'BMI'. The titles and abstracts of studies were screened, and only studies which appeared to match the pre-determined inclusion and exclusion criteria were extracted. The full texts of the extracted studies were read to determine relevance to the current study. To supplement the electronic searches, we also examined the reference list of included studies.

\section{Selection of studies}

Two authors ( $\mathrm{KH}$ and $\mathrm{JHXL}$ ) selected the studies, and differences were resolved by discussion. The inclusion criteria were: (1) studies which identified adult patients diagnosed with COVID-19 based on a real-time reverse transcription PCR method who were hospitalised in an acute hospital, (2) studies which clearly defined the obese and non-obese populations based on BMI, (3) studies which appropriately defined obesity as BMI $>30$ or $>28$, in non-Asians and Asians, respectively, (4) studies which used pre-specified criteria for outcomes of severe COVID-19 illness or mortality, (5) cohort studies which may be prospective or retrospective in nature, and (6) studies with an appropriate control group allowing estimation of odds ratio of unfavourable COVID-19 outcomes between the obese and non-obese groups. If there was suggestion of multiple publications from the same or overlapping group of patients (e. g., studies arising from the same hospital database), we decided to include the data only from the most comprehensive study.

We used the following exclusion criteria: (1) studies which were not in English, (2) studies which focused only on a specific cohort of patients (e. g., only patients in ICU, only patients with diabetes, only geriatric patients), (3) studies which included < 10 patients, (4) studies whose population are not an inpatient cohort, or (5) studies which focused on a paediatric or pregnant population.

\section{Extraction of data}

The data from the included studies were independently extracted and collated on a standardised form by three authors (CWSH, HK, and JHXL). CWSH examined the data for any error. The following data were collated from each study: the first author, the country and city, type of study, time period, location, definitions of obese and non-obese categories, criteria for defining severe COVID-19 illness, clinical and demographic characteristics, and outcomes of patients. Any reported results of univariate or multivariate analysis of outcomes in obese versus non-obese group, as well as the confounders adjusted for, were also collected.

\section{Assessment of quality of the included studies}

Two authors (JHXL and IH) were blinded to the study results and independently assessed the quality of studies using the Newcastle-Ottawa scale [24]. Differences were resolved through discussion. This well-established scale assesses each study across three categories: selection (4 items, maximum 4 stars), comparability ( 1 item, maximum 2 stars) and exposure ( 3 items, maximum 3 stars). Each study receives a total score ranging between 0 and 9 . Scores of $\geq 7,5-6$, and $\leq 4$ translate into 'high', 'medium', and 'low' quality scores respectively.

\section{Aim of study}

The primary outcome of interest was the pooled odds ratio (OR) in obese compared to non-obese hospitalised patients with COVID19 , for the composite outcome of an unfavourable clinical outcome, defined by mortality and, or severe disease, inclusive of:

1) Requiring intensive (ICU) or high-dependency care, or

2) Requiring mechanical ventilation,

3) Categorised as suffering severe pneumonia or acute respiratory distress syndrome (ARDS) as defined by either the WHO, American Thoracic Society (ATS), or National Health Commission of the People's Republic of China criteria [25-27], or

4) Clinician-defined severe disease.

The secondary outcomes of interest were mortality, and severe disease. Among studies which reported outcomes for normal BMI (defined as $\mathrm{BMI}<25$ or $<23.5$ in non-Asian and Asian studies, respectively), overweight and obese categories, we examined the effect of increasing BMI categories on developing an unfavourable outcome, as well as the OR of severe obesity, defined by BMI > 35 or $>40$ on an unfavourable outcome.

Subgroup analyses were done to identify study characteristics that significantly influenced the pooled OR estimate of unfavourable outcome and contributed to heterogeneity between studies. Our pre-determined subgroup analyses of study characteristics compared unfavourable outcomes in studies by:

1) Study region (Asian vs. non-Asian),

2) Quality of study (high, medium or low),

3) Type of study (prospective vs retrospective),

4) Multi-centre versus single-centre, 
5) Sample size of obese population (above vs. below 100 participants), and

6) Prevalence * of possible confounding patient demographics and comorbidities including:

a) Population mean age above versus below 60 years,

b) Prevalence of diabetes mellitus above versus below $30 \%$,

c) Prevalence of hypertension above versus below $60 \%$,

d) Prevalence of CVD above versus below $20 \%$,

e) Prevalence of chronic kidney disease (CKD) above versus below $15 \%$, and

f) Prevalence of chronic pulmonary disease above versus below $20 \%$

* These cut-offs were chosen based on the median of the prevalence reported in all the studies.

\section{Statistical analysis}

For each study, the data for the unfavourable COVID-19 outcomes was reported as the Odds Ratio. Given the expected heterogeneity in the effect sizes, we decided to perform the meta-analysis using a random-effects model [28] based upon the method described by DerSimonian and Laird [29]. The heterogeneity among the studies was assessed using two methods [30]: (a) Cochran's Q statistic which takes into account the overall variance of effect sizes, with subsequent assessment of statistical significance of such heterogeneity (as the tests for heterogeneity have low power, a p-value of $<0.10$ was taken as suggestive of significant heterogeneity); (b) the inconsistency index $\left(\mathrm{I}^{2}\right)$ which depicts the proportion of true heterogeneity among studies from the overall heterogeneity (conventionally, the $\mathrm{I}^{2}$ values of $<30 \%, 30-59 \%, 60-75 \%$ and $>75 \%$ are considered to represent low, moderate, substantial and considerable heterogeneity, respectively). Using the Remove-One index, sensitivity analysis was performed to examine any disproportionate effect of a particular study on the overall estimate of the pooled estimate. Publication bias was ascertained quantitatively using Egger's regression test and qualitatively using funnel plots [31, 32]. If publication bias was found, impact of publication bias was assessed using Duval and Tweedie's "Trim and Fill" method while the 'fail-safe N' test was utilised to estimate the number of unpublished studies with non-significant results that would be required to make the publication bias non-significant $[33,34]$. All analyses were performed using Comprehensive Meta-analysis (CMA) software, version 3 (Biostat Inc., Englewood, NJ, USA).

\section{Results}

\section{Results of literature search}

Initial search yielded 999 articles, and 997 articles remained after removal of duplicates. A total of 819 articles were removed after screening the title and abstract for relevance. A sum of 178 full-text articles were selected and evaluated in detail for potential inclusion, with a further 158 excluded for reasons specified in $\mathbf{F i g . ~} 1$. The remaining 20 studies were used in the qualitative and quantitative meta-analysis.

\section{Characteristics of included studies}

This meta-analysis included 20 studies consisting of a total of 28 355 patients who were hospitalised with COVID-19 illness. The period of hospitalisation was between January and May 2020 ( $\triangleright$ Table 1). All included studies were observational cohort studies, of which 15 were retrospective and 5 were prospective studies. Most studies $(n=11)$ were from the United States, while 5 were from Europe, 3 from Asia (China), and 1 from South America (Mexico). Majority of studies ( $n=12$ ) were based on single centre while 8 were multicentre. Besides two studies which defined obesity as $\mathrm{BMI}>28 \mathrm{~kg} / \mathrm{m}^{2}$, all other studies used the criteria established by the $\mathrm{WHO}$ with respect to BMI.

\section{Quality of studies}

As assessed with the Newcastle Ottawa scale, overall quality of studies was moderate to high. Six studies were of high quality, twelve were of moderate quality, while only two were of low quality. Majority of the studies scored low in the 'comparability' domain of the scale (Supplementary Table 1S).

\section{Population characteristics}

All studies were conducted in adults. The patient characteristics in each study are summarised in Supplementary Table 2S. The mean age of participants was 66.4 (SD 19.5) years, with a predominance of male patients (59.5\%). A total of 6361 (22.4\%) of patients were obese, defined as a BMI above 30 (or above 28 in Asians). Overall, 8097 (28.4\%) patients had an unfavourable outcome. $30.6 \%$ of obese patients had an unfavourable outcome, compared with $28.0 \%$ of non-obese patients.

\section{Risk of unfavourable outcome in obese patients}

As the primary outcome of this study, OR of unfavourable outcome was estimated for obese patients as compared to non-obese patients who were hospitalised with COVID-19 illness. The unadjusted pooled OR 1.25 ( $n=28355$ patients in 9 studies, $95 \% \mathrm{Cl} 1.07-$ $1.45, p=0.005 ; I^{2}=65.8 \%$ ) for an unfavourable outcome ( $\triangleright$ Fig. 2 ). When adjusted for potential confounders, the pooled OR was 2.02 $(\mathrm{n}=17861$ patients in 6 studies, $95 \% \mathrm{Cl} 1.41-2.89, \mathrm{p}<0.001$; $I^{2}=73.5 \%$ ). For both ORs, there were significant and substantial heterogeneity among the studies (discussed later).

\section{Risk of mortality in obese patients}

In the four studies ( $\mathrm{n}=17322$ patients), the adjusted pooled OR for mortality was significantly higher in the obese cohort (OR 1.51, $95 \% \mathrm{Cl} 1.13-2.21, \mathrm{p}=0.006, \mathrm{I}^{2}=46.2 \%$ ) (Supplementary Fig. 1S). The heterogeneity among studies (Cochran's Q 5.48, $p=0.134$ ) and publication bias (Egger's test, $\mathrm{p}=0.362$ ) were non-significant for this outcome.

\section{Risk of severe COVID-19 illness in obese patients}

Four studies consisting of 1547 patients reported severity outcomes with adjustment for age, sex and other comorbidities. The pooled adjusted OR for risk of severe disease in obese versus nonobese patients was 2.26 (95\% Cl 1.47-3.48, p<0.001; $\left.\left.\right|^{2}=67.2 \%\right)$ (Supplementary Fig. 1S). Publication bias was non-significant on Egger's test $(p=0.053)$. 

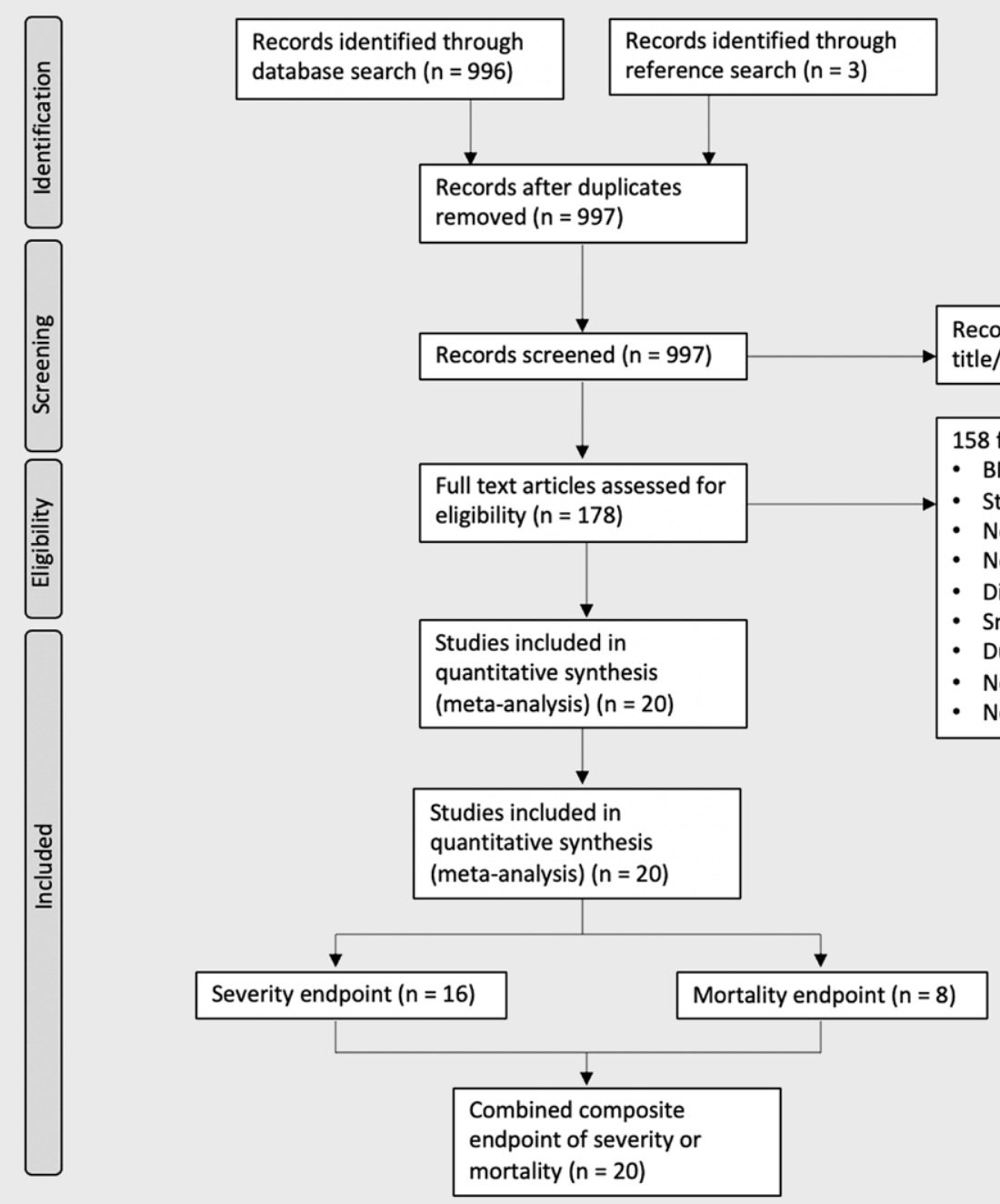

- Fig. 1 PRISMA flow chart of study selection.

\section{Graded analysis between increasing BMI groups and risk of unfavourable outcome, compared to a normal BMI}

Nine studies of 3255 patients reported unfavourable outcomes in patients with normal BMI, overweight and obese groups. Normal weight was defined as $\mathrm{BMI}<25 \mathrm{~kg} / \mathrm{m}^{2}$, overweight was defined as BMI $25-29.9 \mathrm{~kg} / \mathrm{m}^{2}$ and obese was defined as BMI>30 kg/m². One study [11] defined normal weight as BMI $18.5-23.9 \mathrm{~kg} / \mathrm{m}^{2}$, overweight as BMI $24-27.9 \mathrm{~kg} / \mathrm{m}^{2}$ and obese as BMI $>28 \mathrm{~kg} / \mathrm{m}^{2}$. The pooled unadjusted OR comparing overweight and obese groups to normal weight group was 1.81 (95\% Cl 1.10-2.98, $\mathrm{p}=0.019$; $\mathrm{I}^{2}=75 \%$ ), and $2.11\left(95 \% \mathrm{Cl} 1.17-3.82, \mathrm{p}=0.014 ; \mathrm{I}^{2}=78.5 \%\right)$, respectively ( $\triangleright$ Fig. 3 ).

In patients with severe obesity (class II obesity, BMI>35) compared to a normal BMI, the pooled unadjusted OR from 3 studies for an unfavourable outcome was significantly increased at 2.54 (95\% Cl 1.20-5.37, $\mathrm{p}=0.015 ; \mathrm{I}^{2}=0 \%$ ). The adjusted $\mathrm{OR}$ for an unfavourable outcome was similarly increased in 4 studies of severe- ly and morbidly obese patients, as compared to patients with a normal BMI (Supplementary Table 3S).

\section{Subgroup analysis}

As mentioned earlier, significant and substantial heterogeneity were noted for the primary outcomes of interest (ORs of unfavourable outcomes in obese hospitalised patients with COVID-19 illness). For the unadjusted OR of unfavourable outcomes, pre-specified subgroup analysis was performed to explore study-related cofactors which could have influenced the outcomes (Supplementary Table 4S).

Reduction in the heterogeneity was noted when studies were separately analysed according to whether they had large or small numbers of obese patients, with smaller studies ( $<100$ obese patients) reporting a significantly greater risk of unfavourable outcome with obesity and larger studies (>100 obese patients) reporting no significant difference in unfavourable outcomes between obese and non-obese participants. There was no difference observed between subgroups when studies were separated into 


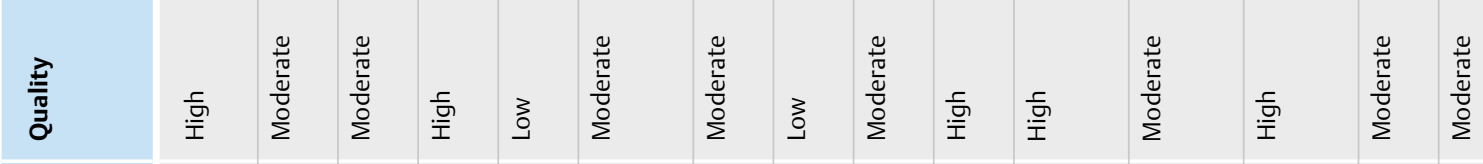

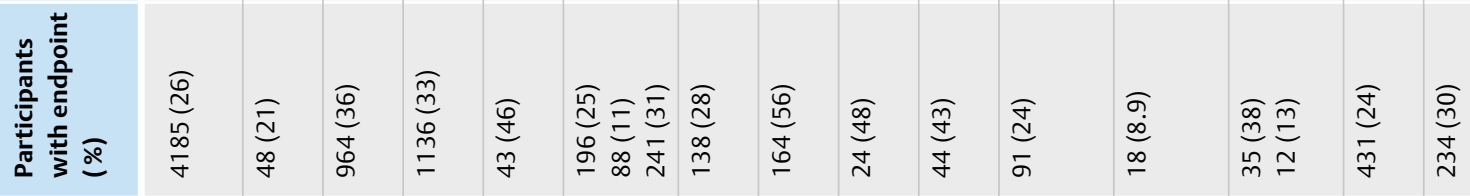

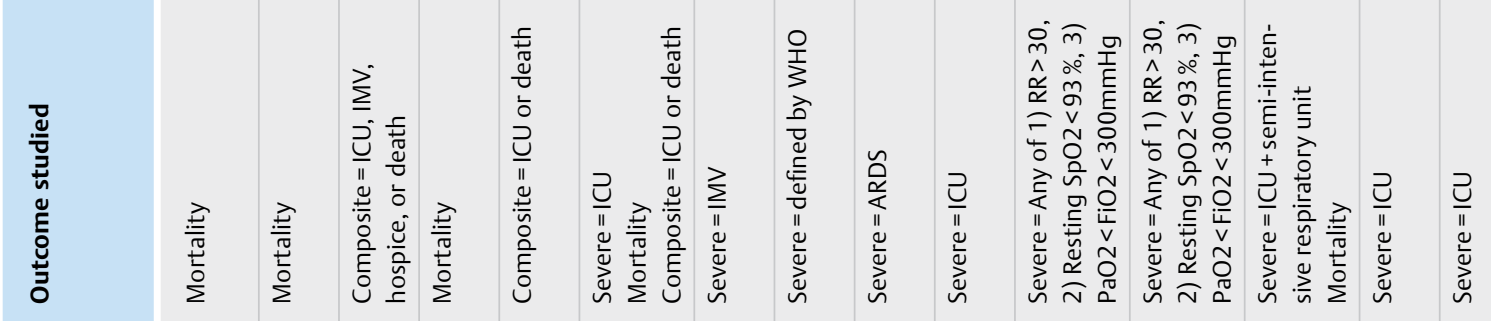

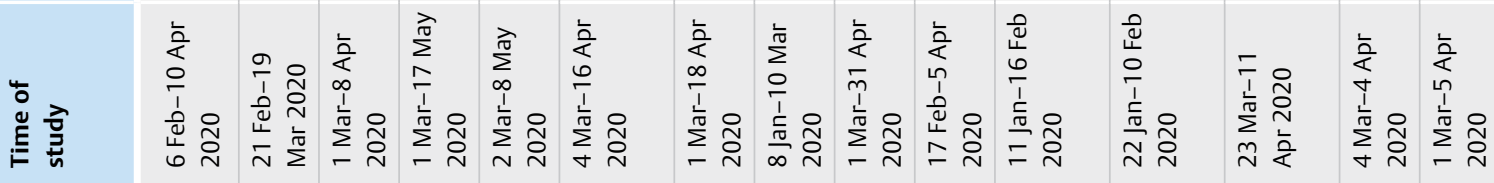

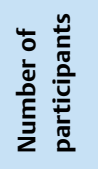

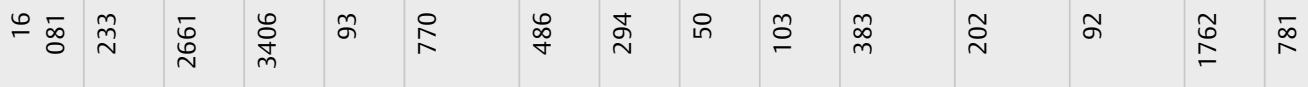

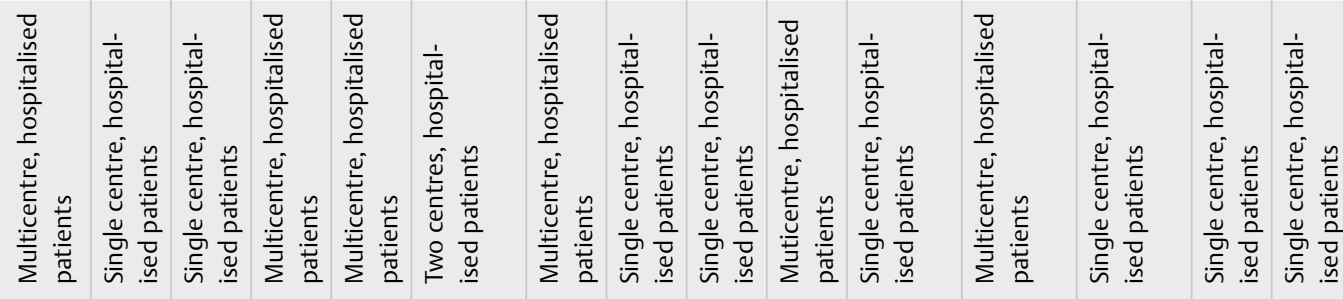
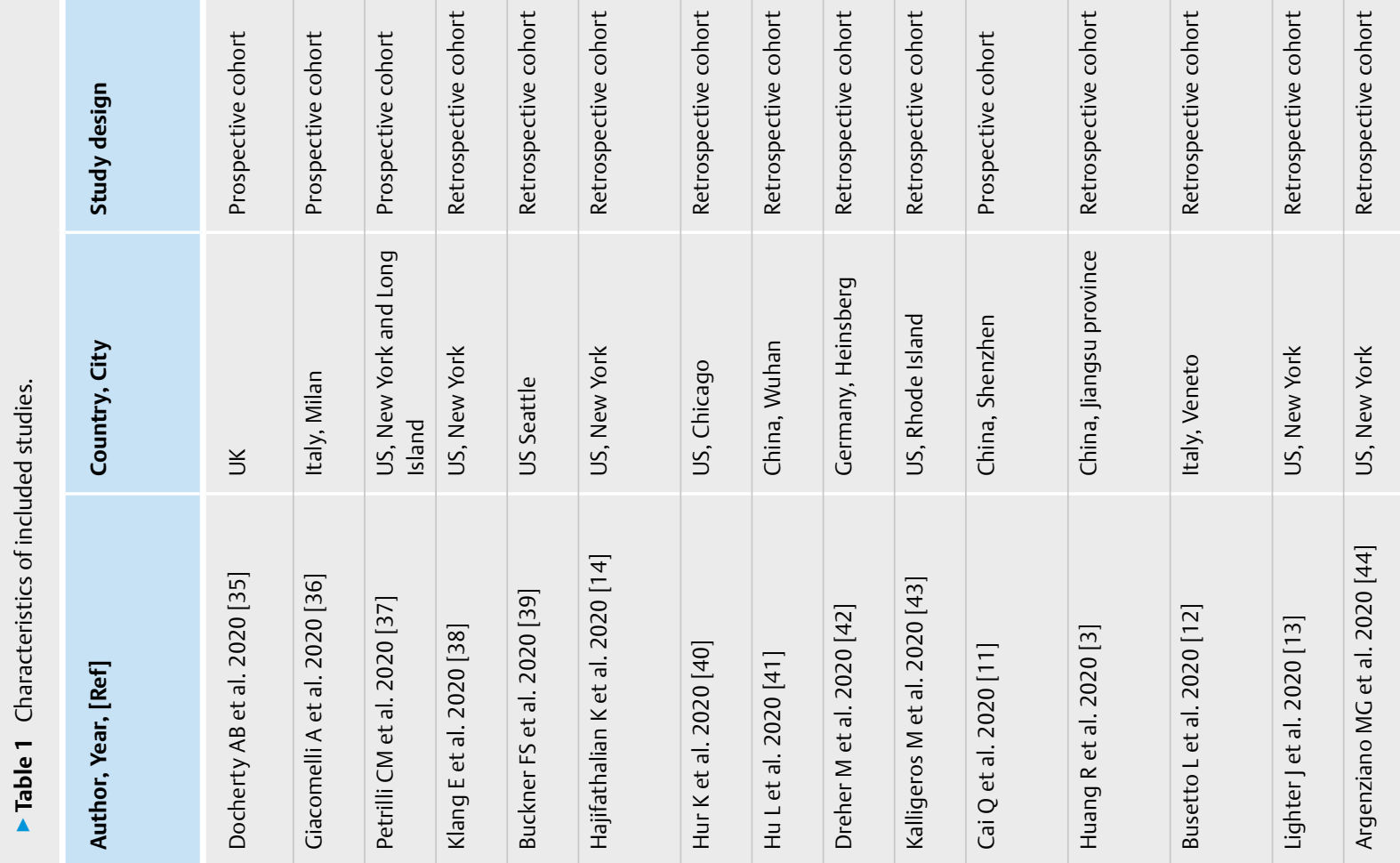


\begin{tabular}{|c|c|c|c|c|c|}
\hline 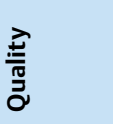 & $\begin{array}{l}\frac{0}{\pi} \\
\frac{0}{4} \\
\frac{0}{0} \\
\Sigma\end{array}$ & 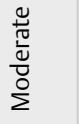 & $\begin{array}{l}\frac{0}{\pi} \\
\frac{0}{2} \\
\frac{0}{0} \\
\Sigma\end{array}$ & 占 & $\begin{array}{l}\frac{0}{\pi} \\
\frac{\pi}{2} \\
\frac{0}{2}\end{array}$ \\
\hline 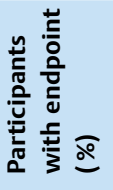 & 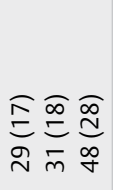 & $\stackrel{\infty}{=}$ & 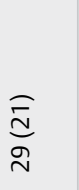 & 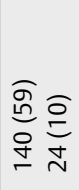 & $\begin{array}{l}\text { 高 } \\
\bar{q} \\
\end{array}$ \\
\hline 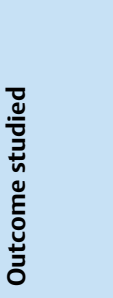 & 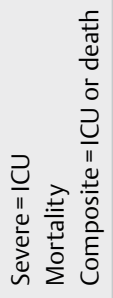 & 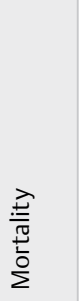 & 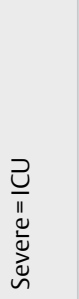 & 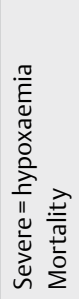 & 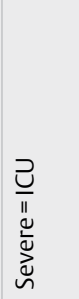 \\
\hline 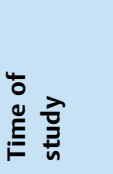 & 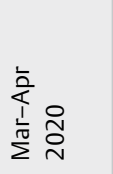 & 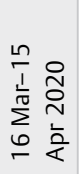 & 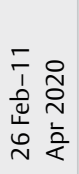 & 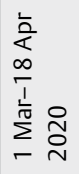 & 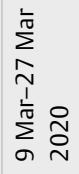 \\
\hline 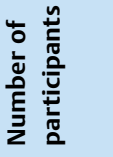 & ㅍ & 음 & 옴 & $\stackrel{\infty}{\sim}$ & $\stackrel{\substack{\text { m } \\
m}}{ }$ \\
\hline : & 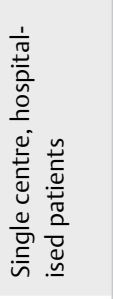 & 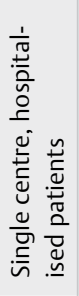 & 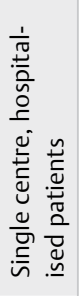 & 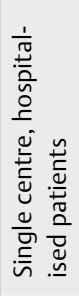 & 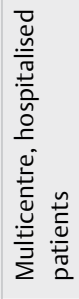 \\
\hline $\begin{array}{l}\frac{0}{\bar{y}} \\
\frac{0}{0} \\
\frac{0}{2} \\
\text { ñ }\end{array}$ & 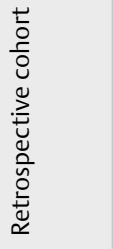 & 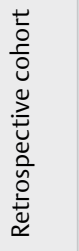 & 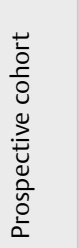 & 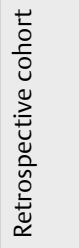 & 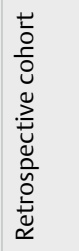 \\
\hline 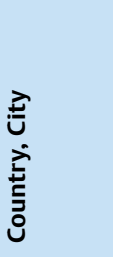 & 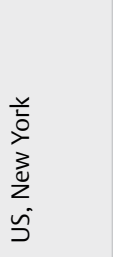 & 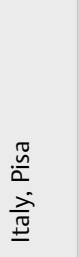 & 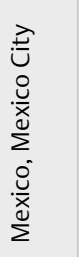 & 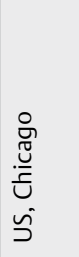 & 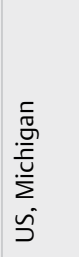 \\
\hline 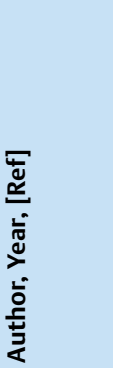 & 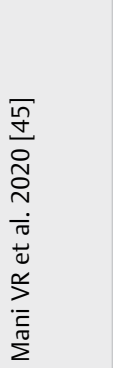 & 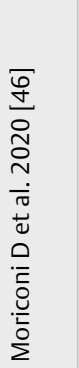 & 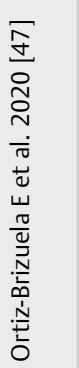 & 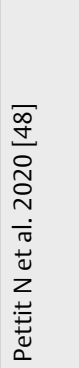 & 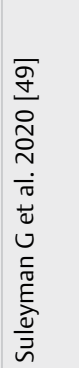 \\
\hline
\end{tabular}

location, multi-centre versus single centre studies, prospective versus retrospective studies, or study quality. Among patient characteristics, a lower prevalence of CVD $<20 \%$ and CKD $<15 \%$ in studies were associated with a higher pooled OR for unfavourable outcome in obese versus non-obese patients.

\section{Sensitivity analysis and publication bias for primary outcomes}

Using Remove-one index for sensitivity analysis, the pooled ORs were re-evaluated after excluding one study at a time. However, no significant change in the heterogeneity or pooled OR was noted, suggesting that our eligibility criteria for inclusion or exclusion of studies did not have an inherent deficiency. Based on the visual inspection of the funnel plot and Egger's test, significant publication bias was found (Supplementary Fig. 2S). For the primary outcome of unfavourable unadjusted $\mathrm{OR}$, the publication bias was re-assessed using the 'Trim and Fill' method of Duvel and Tweedie [33]. Despite trimming five of 20 studies (on the left of the mean), publication bias still remained significant.

\section{Discussion}

To the best of our knowledge, this is the first systematic review and meta-analysis studying an association between obesity and unfavourable outcomes in patients who are hospitalised with COVID19 illness. In this meta-analysis of more than 28000 adult COVID19 infected patients, the risk of an unfavourable outcome (of developing severe disease or dying from COVID-19) was estimated to be approximately twice as compared to the non-obese patients even after adjustment for other potential confounders. Also, obese patients also had $50 \%$ greater odds of mortality compared to nonobese patients. The association of an unfavourable outcome were seen even in patients with BMI in the overweight category, as opposed to a normal BMI, and this was further increased in the severely obese group. This strengthens the relationship between increasing BMI and COVID-19 outcomes observed.

These findings are consistent with the increased risk observed in obese patients for developing critical disease in acute respiratory and non-respiratory illnesses [20, 50,51]. Increased adiposity has been proposed to exacerbate a proinflammatory phenotype by increasing proinflammatory adipokines and reducing anti-inflammatory adipokines [52], resulting in a chronic inflammatory process that involves tonic activation of the innate immune system [53]. Obesity also results in mechanical compression of the thorax, including the chest walls, lungs, and diaphragm [54], and has been shown to have detrimental effects to respiratory function in all age groups [55].

As a known risk factor for diabetes and cardiovascular disease which are independently associated with morbidity and mortality $[56,57]$, obesity may also be associated with poorer overall health outcomes. However, studies have not been consistent. The obesity paradox has been described in which obesity seems to be a protective factor in patients with established CV disease and advanced CKD $[58,59]$. This appears to be consistent with our subgroup analysis showing a lower OR of obesity for an unfavourable outcome in populations with a higher prevalence of CV disease and CKD. 


\begin{tabular}{|c|c|c|c|c|}
\hline \multirow[t]{2}{*}{ Study name } & \multicolumn{4}{|c|}{ Statistics for each study } \\
\hline & $\begin{array}{l}\text { Odds } \\
\text { ratio }\end{array}$ & $\begin{array}{l}\text { Lower } \\
\text { limit }\end{array}$ & $\begin{array}{c}\text { Upper } \\
\text { limit }\end{array}$ & $\mathrm{p}-\mathrm{Va}$ \\
\hline${ }^{a}$ Docherty AB & 0.928 & 0.825 & 1.042 & \\
\hline Giacomelli A & 2.377 & 1.108 & 5.101 & 0.0 \\
\hline Petrilli CM & 0.888 & 0.755 & 1.043 & \\
\hline Klang E & 0.858 & 0.739 & 0.996 & \\
\hline Buckner FS & 1.588 & 0.699 & 3.610 & 0.2 \\
\hline Hajifathalian $\mathrm{K}$ & 1.340 & 0.979 & 1.834 & \\
\hline Hur K & 1.475 & 0.988 & 2.201 & \\
\hline $\mathrm{Hu} \mathrm{L}$ & 1.282 & 0.409 & 4.016 & 0.6 \\
\hline Dreher M & 2.821 & 0.837 & 9.509 & \\
\hline Kalligeros $\mathrm{M}$ & 1.919 & 0.870 & 4.232 & \\
\hline Cai Q & 2.142 & 1.087 & 4.221 & \\
\hline Huang $\mathrm{R}$ & 6.900 & 2.381 & 19.997 & \\
\hline Busetto L & 1.228 & 0.499 & 3.018 & \\
\hline Lighter J & 1.264 & 1.016 & 1.573 & \\
\hline Argenziano MG & 1.291 & 0.948 & 1.759 & \\
\hline Mani VR & 1.059 & 0.535 & 2.098 & \\
\hline Moroconi D & 0.929 & 0.299 & 2.894 & \\
\hline Ortiz-Brizuela E & 2.327 & 1.013 & 5.342 & \\
\hline Pettit NN & 0.847 & 0.474 & 1.515 & \\
\hline Suleyman G & 1.192 & 0.772 & 1.841 & \\
\hline Total & 1.245 & 1.069 & 1.449 & \\
\hline b Docherty AB & 1.330 & 1.189 & 1.488 & \\
\hline Giacomelli A & 3.040 & 1.422 & 6.499 & \\
\hline Hajifathalian K & 1.580 & 1.176 & 2.123 & \\
\hline Cai $Q$ & 3.400 & 1.400 & 8.259 & \\
\hline Huang R & 9.219 & 2.731 & 31.123 & \\
\hline Pettit NN & 1.700 & 0.964 & 2.999 & \\
\hline Total & 2.019 & 1.410 & 2.892 & \\
\hline
\end{tabular}

Odds ratio and $95 \% \mathrm{CI}$

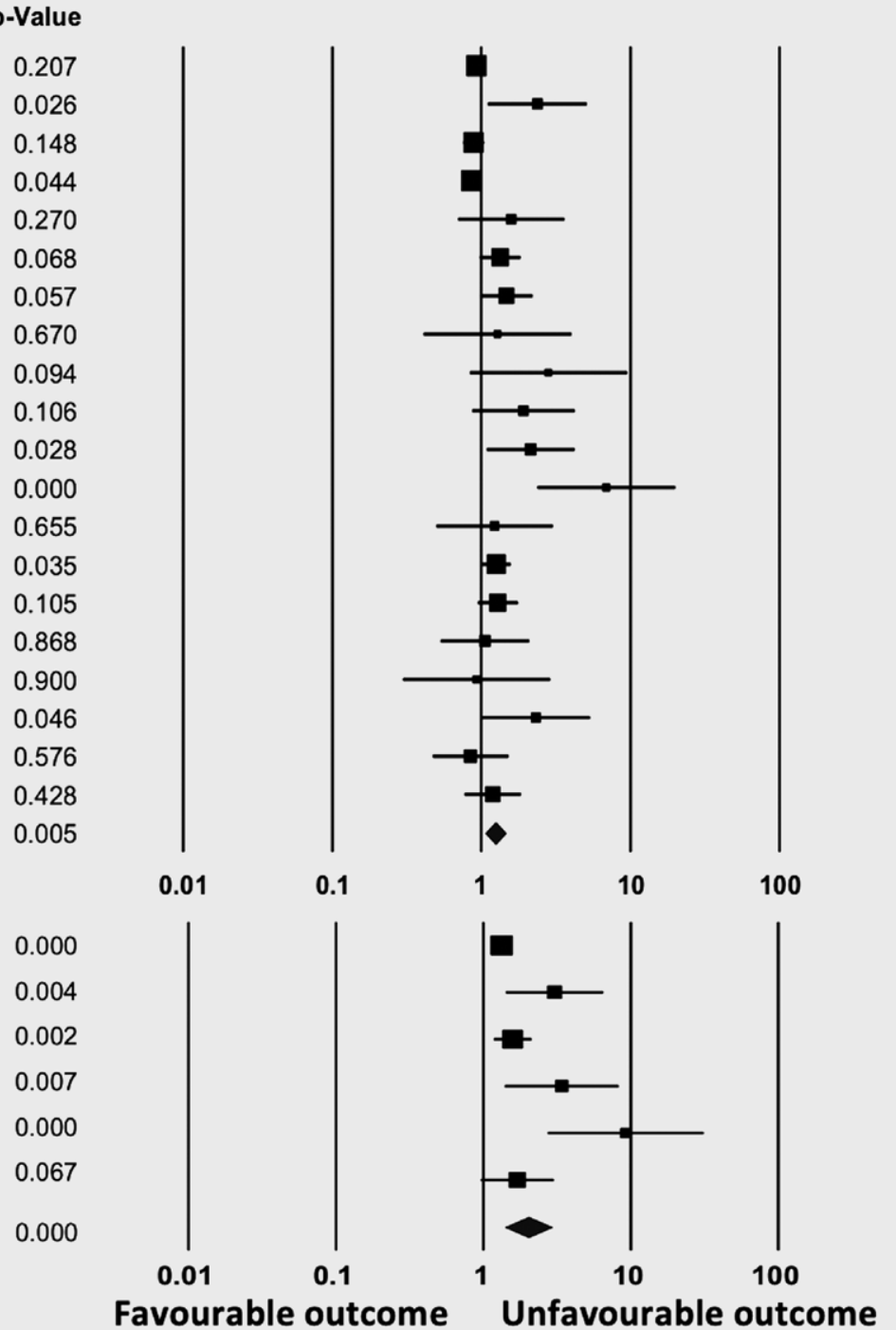

- Fig. 2 Forrest plot showing a significantly increased pooled odds ratio for obese compared to non-obese patients for an unfavourable outcome, including studies reporting severity and mortality. a Unadjusted odds ratio. $\mathbf{b}$ Adjusted odds ratio for age, sex and major co-morbidities.

There are several mentionable strengths in our study: systematic strategy for search of the literature with well-defined criteria for inclusion and exclusion; appropriate exclusion of redundant and non-informative studies, meticulous extraction of data (both explicit as well as deduced from the studies), careful evaluation for quality of studies, and appropriate quantitative statistical assessment. Based on the large sample size in the included studies from different geographical locations of the globe, our estimate appears to have enough power (i. e., low risk for type Il error) for a comfortable acceptance. Moreover, our estimates were carefully calculated with and without adjustment for potential confounders, thereby providing a better understanding of the impact of obesity on the unfavourable outcomes in patients with COVID-19 illness.

We also acknowledge several limitations in our study. First, majority of the studies were retrospective in nature which might have introduced intrinsic biases (e. g., recall bias, reporting bias, etc.). Secondly, our population for the systematic review was only hospitalised patients with COVID-19 illness. As the criteria for hospitalisation may differ between hospitals, the population of included studies might not have been uniform. To explore it further, we carefully performed a subgroup analysis for a variety of study and patient related factors. The only partial explanation for heterogeneity was that there was a significant difference in estimates in studies with larger and smaller samples. Moreover, the sensitivity analysis did not alter the estimate of pooled OR thereby providing weight to the robustness of our eligibility criteria. We believe that most of the heterogeneity could have arisen from having different types of hospitalisations (such as varying criteria for hospitalisation, escalation of treatment or definition of severity). 


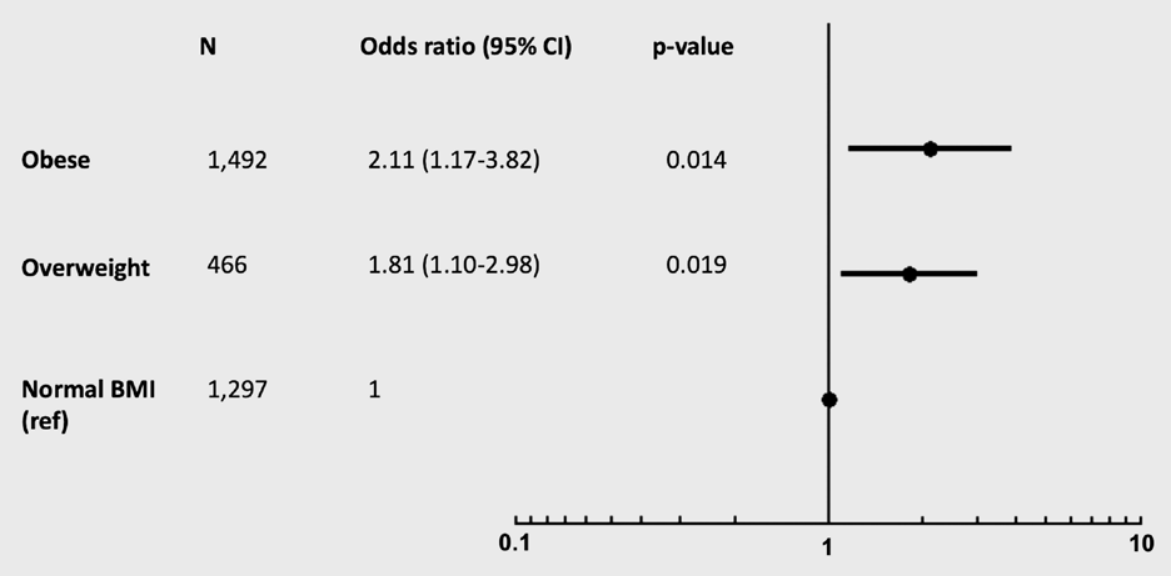

- Fig. 3 Pooled unadjusted OR of 9 studies reporting unfavourable outcome in overweight and obese COVID-19 patients, as compared to normal weight patients. $\mathrm{N}=$ total number of participants.

\section{Conclusion}

Based on a large sample, our systematic review demonstrates that obesity significantly and independently increases the odds of an unfavourable outcome in hospitalised patients with COVID-19 illness. In addition, obesity is associated with increased mortality and severe course of illness. Risk stratification and a review of resource allocation for the obese patient with COVID-19 is pivotal to providing optimal care in this population. Given the growing epidemic of COVID-19 and the high prevalence of obesity globally, there is a pressing need for population strategies aimed to prevent and manage obesity, and it remains to be seen if these can be helpful in reducing overall morbidity and mortality in COVID-19.

\section{Conflict of Interest}

The authors declare that they have no conflict of interest.

\section{References}

[1] World Health Organization. Statement on the second meeting of the International Health Regulations (2005) Emergency Committee regarding the outbreak of novel coronavirus (2019-nCoV); https:// www.who.int/news-room/detail/30-01-2020-statement-on-the-second-meeting-of-the-international-health-regulations-(2005)-emergency-committee-regarding-the-outbreak-of-novel-coronavirus-(2019-ncov) (Assessed 7 Jul 2020)

[2] Worldometer. Coronavirus Update (Live): 11603648 Cases and 537707 Deaths from COVID-19 Virus Pandemic; https://www.worldometers. info/coronavirus/ (Assessed 7 Jul 2020)

[3] Huang C, Wang Y, Li X et al. Clinical features of patients infected with 2019 novel coronavirus in Wuhan, China. Lancet 2020; 395: 497-506

[4] Wang D, Hu B, Hu C et al. Clinical characteristics of 138 hospitalized patients with 2019 novel coronavirus-infected pneumonia in Wuhan, China. JAMA 2020; 323: 1061-1069

[5] Deng Y, Liu W, Liu K et al. Clinical characteristics of fatal and recovered cases of coronavirus disease 2019 in Wuhan, China: A retrospective study. Chin Med J 2020; 133: 1261-1267
[6] Chen N, Zhou M, Dong X et al. Epidemiological and clinical characteristics of 99 cases of 2019 novel coronavirus pneumonia in Wuhan, China: A descriptive study. Lancet 2020; 395: 507-513

[7] World Health Organisation COVID-19 High risk groups. https://www. who.int/westernpacific/emergencies/covid-19/information/ high-risk-groups (Assessed 7 Jul 2020)

[8] Centers for Disease Control and Prevention Coronavirus Disease 2019 (COVID-19): People of any age with underlying medical conditions. https://www.cdc.gov/coronavirus/2019-ncov/need-extra-precautions/ people-with-medical-conditions.html (Assessed 7 Jul 2020)

[9] NHS Digital Coronavirus (COVID-19): Shielded patients list. https:// digital.nhs.uk/coronavirus/shielded-patient-list (Assessed 7 Jul 2020)

[10] Ministry of Health Singapore Advisory on vulnerable group. https:// www.moh.gov.sg/docs/librariesprovider5/advisories/advisory-on-vulnerable-group-(moh).pdf (Assessed 7 Jul 2020)

[11] Cai Q, Chen F, Wang T et al. Obesity and COVID-19 severity in a designated hospital in Shenzhen, China. Diabetes Care 2020; 43: 1392-1398

[12] Busetto L, Bettini S, Fabris R et al. Obesity and COVID-19: An Italian snapshot. Obesity (Silver Spring) 2020; 28: 1600-1605

[13] Lighter J, Phillips M, Hochman S et al. Obesity in patients younger than 60 years is a risk factor for Covid-19 hospital admission. Clin Infect Dis 2020; 71: 896-897

[14] Hajifathalian K, Kumar S, Newberry C et al. Obesity is associated with worse outcomes in COVID-19: Analysis of Early Data From New York City. Obesity (Silver Spring) 2020; 28: 1606-1612

[15] Segaloff HE, Evans R, Arshad S et al. The impact of obesity and timely antiviral administration on severe influenza outcomes among hospitalized adults. J Med Virol 2018; 90: 212-218

[16] Myles PR, Semple MG, Lim WS et al. Predictors of clinical outcome in a national hospitalised cohort across both waves of the influenza A/H1N1 pandemic 2009-2010 in the UK. Thorax 2012; 67: 709-717

[17] Riquelme R, Jiménez P, Videla AJ et al. Predicting mortality in hospitalized patients with $2009 \mathrm{H} 1 \mathrm{~N} 1$ influenza pneumonia. Int J Tuberc Lung Dis 2011; 15: 542-546

[18] Morgan OW, Bramley A, Fowlkes A et al. Morbid obesity as a risk factor for hospitalization and death due to 2009 pandemic influenza $A(H 1 N 1)$ disease. PLoS One 2010; 5: e9694

[19] Bassetti M, Parisini A, Calzi A et al. Risk factors for severe complications of the novel influenza A (H1N1): Analysis of patients hospitalized in Italy. Clin Microbiol Infect 2011; 17: 247-250 
[20] Mertz D, Kim TH, Johnstone J et al. Populations at risk for severe or complicated influenza illness: Systematic review and meta-analysis. BMJ 2013; 347: f5061

[21] Higgins JPT, Thomas J, Cumpston M et al. Cochrane Handbook for Systematic Reviews of Interventions. second edition. Chichester (UK): John Wiley \& Sons; 2019

[22] Moher D, Liberati A, Tetzlaff J et al. Preferred reporting items for systematic reviews and meta-analyses: The PRISMA statement. Ann Intern Med 2009; 151: 264-269 W64

[23] Stroup DF, Berlin JA, Morton SC et al. Meta-analysis of observational studies in epidemiology: a proposal for reporting. Meta-analysis Of Observational Studies in Epidemiology (MOOSE) group. JAMA 2000; 283: 2008-2012

[24] Stang A. Critical evaluation of the Newcastle-Ottawa scale for the assessment of the quality of nonrandomized studies in meta-analyses. Eur J Epidemiol 2010; 25: 603-605

[25] World Health Organization Clinical management of COVID-19. https://www.who.int/publications-detail-redirect/clinical-managementof-covid-19 (Assessed 7 Jul 2020)

[26] Fan E, Del Sorbo L, Goligher EC et al. An Official American Thoracic Society/European Society of Intensive Care Medicine/Society of Critical Care Medicine Clinical Practice Guideline: Mechanical Ventilation in Adult Patients with Acute Respiratory Distress Syndrome. Am J Respir Crit Care Med 2017; 195: 1253-1263

[27] National Health Commission Guidelines for the Diagnosis and Treatment of Novel Coronavirus (2019-nCoV) Infection by the National Health Commission (Trial Version 5). http://www.nhc.gov.cn/yzygj/ s7653p/202002/3b09b894ac9b4204a79db5b8912d4440.shtml (Assessed 7 Jul 2020)

[28] Riley RD, Higgins JPT, Deeks J]. Interpretation of random effects meta-analyses. BMJ 2011; 342: d549

[29] DerSimonian R, Laird N. Meta-analysis in clinical trials revisited. Contemp Clin Trials 2015; 45: 139-145

[30] Higgins JPT, Thompson SG, Deeks ]J et al. Measuring inconsistency in meta-analyses. BMJ 2003; 327: 557-560

[31] Sterne JAC, Sutton AJ, loannidis JPA et al. Recommendations for examining and interpreting funnel plot asymmetry in meta-analyses of randomised controlled trials. BM] 2011; 343: d4002

[32] Easterbrook PJ, Gopalan R, Berlin JA et al. Publication bias in clinical research. Lancet 1991; 337: 867-872

[33] Duval S, Tweedie R. Trim and fill: A simple funnel-plot-based method of testing and adjusting for publication bias in meta-analysis. Biometrics 2000; 56: 455-463

[34] Rosenthal R. The file drawer problem and tolerance for null results. Psychol Bull 1979; 638-641

[35] Docherty AB, Harrison EM, Green CA et al. Features of 20133 UK patients in hospital with covid-19 using the ISARIC WHO Clinical Characterisation Protocol: prospective observational cohort study. BM] 2020; 369: m1985

[36] Giacomelli A, Ridolfo AL, Milazzo L et al. 30-day mortality in patients hospitalized with COVID-19 during the first wave of the Italian epidemic: A prospective cohort study. Pharmacol Res 2020; 158: 104931

[37] Petrilli CM, Jones SA, Yang J et al. Factors associated with hospital admission and critical illness among 5279 people with coronavirus disease 2019 in New York City: prospective cohort study. BMJ 2020; 369: m1966

[38] Klang E, Kassim G, Soffer S et al. Morbid obesity as an independent risk factor for COVID-19 mortality in hospitalized patients younger than 50. Obesity (Silver Spring) 2020; 28: 1595-1599

[39] Buckner FS, McCulloch DJ, Atluri V et al. Clinical features and outcomes of 105 hospitalized patients with COVID-19 in Seattle, Washington. Clin Infect Dis 2020; 71: 2167-2173
[40] Hur K, Price CPE, Gray EL et al. Factors associated with intubation and prolonged intubation in hospitalized patients with COVID-19. Otolaryngol Head Neck Surg 2020; 163: 170-178

[41] Hu L, Chen S, Fu Y et al. Risk factors associated with clinical outcomes in 323 COVID-19 hospitalized patients in Wuhan, China. Clin Infect Dis 2020; 19: 71 2089-2098

[42] Dreher M, Kersten A, Bickenbach J et al. The Characteristics of 50 Hospitalized COVID-19 patients with and without ARDS. Dtsch Arztebl Int 2020; 117: 271-278

[43] Kalligeros M, Shehadeh F, Mylona EK et al. Association of obesity with disease severity among patients with COVID-19. Obesity (Silver Spring) 2020; 28: 1200-1204

[44] Argenziano MG, Bruce SL, Slater CL et al. Characterization and clinical course of 1000 patients with coronavirus disease 2019 in New York: retrospective case series. BMJ 2020; 369: m1996

[45] Mani VR, Kalabin A, Valdivieso SC et al. At the epicenter of the American Coronavirus outbreak - New York inner city hospital COVID-19 experience and current data: A retrospective analysis. J Med Internet Res 2020; 22: e20548

[46] Moriconi D, Masi S, Rebelos E et al. Obesity prolongs the hospital stay in patients affected by COVID-19, and may impact on SARS-COV-2 shedding. Obes Res Clin Pract 2020; 14: 205-209

[47] Ortiz-Brizuela E, Villanueva-Reza M, González-Lara MF et al. Clinical and epidemiological characteristics of patients diagnosed with covid-19 in a tertiary care center in Mexico City: A prospective cohort study. Rev Invest Clin 2020; 72: 165-177

[48] Pettit NN, MacKenzie EL, Ridgway J et al. Obesity is associated with increased risk for mortality among hospitalized patients with COVID-19. Obesity (Silver Spring) 2020; 28: 1806-1810

[49] Suleyman G, Fadel RA, Malette KM et al. Clinical characteristics and morbidity associated with coronavirus disease 2019 in a series of patients in Metropolitan Detroit. JAMA Netw Open 2020; 3: e2012270

[50] Sakr Y, Madl C, Filipescu D et al. Obesity is associated with increased morbidity but not mortality in critically ill patients. Intensive Care Med 2008; 34: 1999

[51] Fezeu L, Julia C, Henegar A et al. Obesity is associated with higher risk of intensive care unit admission and death in influenza $A(H 1 N 1)$ patients: a systematic review and meta-analysis. Obes Rev 2011; 12 : 653-659

[52] Mancuso P. Obesity and respiratory infections: does excess adiposity weigh down host defense? Pulm Pharmacol Ther 2013; 26: 412-419

[53] Saltiel AR, Olefsky JM. Inflammatory mechanisms linking obesity and metabolic disease. J Clin Invest 2017; 127: 1-4

[54] Mafort TT, Rufino R, Costa CH et al. Obesity: systemic and pulmonary complications, biochemical abnormalities, and impairment of lung function. Multidiscip Respir Med 2016; 11: 28

[55] Forno E, Han Y-Y, Mullen J et al. Overweight, obesity, and lung function in children and adults - a meta-analysis. J Allergy Clin Immunol Pract 2018; 6: 570-581.e10

[56] Riaz H, Khan MS, Siddiqi T] et al. Association between obesity and cardiovascular outcomes: A systematic review and meta-analysis of Mendelian Randomization Studies. JAMA Netw Open 2018; 1 : e183788

[57] Zoppini G, Fedeli U, Schievano E et al. Mortality from infectious diseases in diabetes. Nutr Metab Cardiovasc Dis 2018; 28: 444-450

[58] Uretsky S, Messerli FH, Bangalore S et al. Obesity paradox in patients with hypertension and coronary artery disease. Am J Med 2007; 120: 863-870

[59] Kalantar-Zadeh K, Rhee CM, Chou J et al. The obesity paradox in kidney disease: How to reconcile it with obesity management. Kidney Int Rep 2017; 2: 271-281 